\title{
Dynamically expressed ELAV is required for learning and memory in bees
}

\section{PINAR USTAOGLU ${ }^{1 *}$, JATINDER KAUR GILL ${ }^{1 *}$, NICOLAS DOUBOVETZKY ${ }^{2}$, IRMGARD U. HAUSSMANN ${ }^{1,3}$, JEAN-MARC DEVAUD ${ }^{2}$ AND MATTHIAS SOLLER ${ }^{1,}$}

1School of Biosciences, College of Life and Environmental Sciences, University of Birmingham,

8 Edgbaston, Birmingham, B15 2TT, United Kingdom

$9 \quad{ }^{2}$ Research Center on Animal Cognition (CRCA), Center for Integrative Biology (CBI), Toulouse

University, CNRS, UPS, Toulouse 31062, France

11 3Department of Life Science, Faculty of Health, Education and Life Sciences, Birmingham City

12 University, Birmingham, B15 3TN, United Kingdom

13 4Birmingham Centre for Genome Biology, University of Birmingham, Edgbaston, Birmingham,

14 B15 2TT, United Kingdom

\section{Running Title: ELAV requirement for learning and memory}

17 Key Words: ELAV/Hu family proteins, alternative splicing/polyadenylation, neuron-specific

18 RNA binding protein, immediate early gene, learning and memory, microexon

$20 *$ equal contributing authors

215 Corresponding author

22 Matthias Soller

23 School of Bioscience

24 College of Life and Environmental Sciences

25 University of Birmingham

26 Edgbaston

27 Birmingham B15 2TT

28

m.soller@bham.ac.uk

Tel \#: 01214145905 


\section{Abstract}

32 Changes in gene expression are a hallmark of learning and memory consolidation. Little is

33 known about how alternative mRNA processing, particularly abundant in neuron-specific genes,

34 contributes to these processes. Prototype RNA binding proteins of the neuronally expressed

35 ELAV/Hu family are candidates for roles in learning and memory, but their capacity to cross-

36 regulate and take over each other's functions complicate substantiation of such links. Therefore,

37 we focused on honey bees, which have only a single elav family gene. We find that honey bee

38 elav contains a microexon, which is evolutionary conserved between invertebrates and humans.

39 RNAi knockdown of elav demonstrates that ELAV is required for learning and memory in bees.

40 Indicative of a role as immediate early gene, ELAV is dynamically expressed with altered

41 alternative splicing and subcellular localization in mushroom bodies, but not in other brain parts.

42 Expression and alternative splicing of elav change during memory consolidation illustrating an

43 alternative mRNA processing program as part of a local gene expression response underlying

44 memory formation. Although the honey bee genome encodes only a single elav gene, functional

45 diversification is achieved by alternative splicing. 


\section{Introduction}

Changes in gene expression play pivotal roles in memory consolidation, the process

51 through which memories are stabilized and stored into long-term memory ${ }^{1-3}$. Among genes

52 induced upon learning are immediate early genes (IEGs) such as transcription factors egr-1, fos

53 and jun, and changes in transcription seems to be a highly conserved requirement for memory

54 formation, including in insects ${ }^{4-10}$. A common feature of neuronal genes, particularly ion

55 channel and cell adhesion genes, is their often complex pattern of alternative splicing, which

56 alters protein coding and regulatory potential in flanking untranslated regions of the mRNA ${ }^{11-13}$.

57 Since very little is known about alternative splicing programs that operate in learning and

58 memory we focused on ELAV (Embryonic Lethal Abnormal Visual system)/Hu family RNA

59 binding proteins because they are prominently expressed in neurons of all metazoans, regulate

60 alternative splicing and expression of synaptic genes as well as formation of new connections ${ }^{14-}$

$611^{18}$.

62 Like many RNA binding proteins (RBPs) ELAV/Hu proteins comprise a family of highly

63 related proteins in most animals including humans. Humans have four ELAV/Hu genes $(\mathrm{HuB}$,

$64 \mathrm{HuC}, \mathrm{HuD}$ and $H u R$ ), while Drosophila have three (elav, fne and Rbp9), which derive from a

65 common ancestor, but have duplicated independently during vertebrate and arthropod evolution

$66{ }^{19}$. In mice, all Hu proteins are expressed in largely overlapping patterns in mature neurons ${ }^{20}$,

67 while in Drosophila pan-neural expression of ELAV and FNE starts with the birth of neurons,

68 and RBP9 is first detected in late larval neurons ${ }^{21-24}$. Although ELAV family RBPs in

69 Drosophila have distinct neuronal phenotypes based on the analysis of null mutants and genetic

70 interactions among them, they can cross regulate each other's targets depending on cellular

71 localization and concentrations complicating the analysis of their functions ${ }^{24}$. 
ELAV/Hu proteins are proto-type RBPs, which harbor three highly conserved RNA

73 Recognition Motifs (RRMs). The first two RRMs are arranged in tandem and the third RRM is

74 separated by a less-conserved hinge region. ELAV/Hu family RBPs bind to short, uridine-rich

75 motives, which are ubiquitously found in introns and untranslated regions, but ELAV/Hu

76 proteins are gene-specific and have a complement of dedicated target genes ${ }^{18,25-27}$. Due to the

77 prominent nuclear localization, ELAV in Drosophila has mostly been associated with gene-

78 specific regulation of alternative splicing and polyadenylation, but it can also regulate mRNA

79 stability ${ }^{28-34}$. Although the three RRMs comprise the evolutionary most conserved parts of

80 ELAV/Hu proteins, individual members are to a large degree functionally interchangeable when

81 adjusting expression levels and sub-cellular localization ${ }^{24,35,36}$. Hence, regulation of the activity

82 of ELAV/Hu proteins likely occurs at the level of post-translational modifications and suggest

83 that less conserved and unstructured linker sequences between or within RRMs serve

84 fundamental functional roles, possibly by regulating interactions with other proteins ${ }^{37}$.

85 To avoid complications of assigning specific gene functions to individual members of the

86 ELAV/Hu family, we focused on honey bees whose genome encodes only one copy of the elav

87 gene ${ }^{19}$, an orthologue of Drosophila fne ${ }^{22}$. Conveniently, honey bees are a well-established

88 model for the study of learning and memory. Here we show that the single elav gene in honey

89 bees is required for learning as well as formation of stable memories by RNAi knockdown.

90 Although bees have only a single elav gene, its coding capacity proliferated by increasing

91 alternative splicing to generate 38 different isoforms. The splicing pattern changes during

92 development and between different adult social castes, but also shows variability among brains

93 of individua adult workers. Likewise, ELAV expression changes in mushroom bodies (brain

94 centers involved in learning and memory), but not in the medulla of the optic system, to generate 
95 individual expression patterns reminiscent of experience dependent neuronal activity that forms

96 the basis of gene expression changes associated with memory consolidation. Hence, ELAV

97 expression resembles that of immediate early genes (IFGs) induced upon different experiences

$98 \quad 38$. Consistent with a role in learning and memory consolidation, elav expression and inclusion

99 levels of alternative exons change during the early phases of memory consolidation that requires

100 transcription ${ }^{10,39}$.

101

102 Results

103 ELAV is required for learning and memory consolidation in bees after olfactory reward

104 conditioning.

105 To assess whether ELAV has a role in learning and memory in bees the single bee elav gene was

106 knocked down by RNAi leading to a reduction of $80 \%$ after two days (n=3, Fig 1A). Two days

107 after injection of elav or GFP control dsRNA, bees were individually trained and short-term

108 memory was scored 2 hours after training (Fig 1B). Both groups showed significant learning

109 over the successive trials (RM-ANOVA, Trial effect: $F=61.93, \mathrm{p}<0.001$ ), but performance was

110 affected by treatment (Trial $\times$ Treatment interaction: $\mathrm{F}=4.33, \mathrm{p}<0.05$ ). Indeed, as compared to

111 controls, significantly fewer elav dsRNA-injected bees showed conditioned responses by the end

112 of training (Fischer's test on $3^{\text {rd }}$ trial: $\chi^{2}=4.22, \mathrm{p}<0.05$, Fig $1 \mathrm{C}$ left). However, short-term

113 memory retrieval remained unaffected $\left(\chi^{2}=0.64, \mathrm{p}>0.05\right.$, Fig $1 \mathrm{C}$ right $)$.

114 We then asked whether elav knockdown might impact on the consolidation of long-term memory

115 independently on its effect on acquisition. Therefore, injections and training were performed as

116 before to ensure that elav levels would still be reduced during the hours following training (Fig

117 1D), i.e. at a time when crucial transcriptional activity is required for long-term memory 
118 consolidation ${ }^{10,39}$. We then tested for their memory two days after training (a typical delay to

119 assess consolidated long-term memory). In these conditions, learning occurred normally (RM-

120 ANOVA, Trial effect: F=108.6, $\mathrm{p}<0.001$; Trial $\times$ Treatment interaction: F=0.50, p>0.05; Fig 1E

121 left). Yet, the two groups showed different capacities to recall the memory of the CS-US

122 association (Fischer's test: $\chi^{2}=10.08, \mathrm{p}<0.01$, Fig $1 \mathrm{E}$ right). In addition, only control bees

123 responded significantly more to the CS than to the novel odorant $\left(G F P: \chi^{2}=11.55, \mathrm{p}<0.001\right.$;

124 elav: $\left.\chi^{2}=3.77, \mathrm{p}>0.05\right)$.

125 To reject the possibility that loss of ELAV impairs long-term memory retrieval per se due to a 126 prolonged downregulation of elav, we performed an additional experiment in which injection

127 was done shortly before training, when RNAi is not yet effective (Fig. 1F). As expected, this 128 treatment did not affect learning (Trial effect: $\mathrm{F}=62.93, \mathrm{p}<0.001$; Trial $\mathrm{x}$ Treatment interaction:

$129 \mathrm{~F}=0.15, \mathrm{p}>0.05$; Fig. $1 \mathrm{G}$ left). More importantly, memory retrieval was intact and two days after

130 training both groups responded similarly to the CS (Fischer's test: $\chi^{2}=0.02, \mathrm{p}>0.05$ ) and

131 responded significantly less to the novel odorant $\left(G F P: \chi^{2}=6.24, \mathrm{p}<0.05\right.$; elav: $\chi^{2}=5.66$,

$132 \mathrm{p}<0.05)$, thus indicating a preserved memory of the CS-US association.

133 These results thus argue that elav is required for the early formation of an associative memory 134 over repeated acquisition trials, and for its subsequent consolidation.

\section{The single bee ELAV gene is dynamically alternatively spliced}

137 The bee ELAV protein is highly homologous to those of the Drosophila ELAV family (ELAV, 138 FNE and RBP9) in the three RRM domains, but diverges significantly in the unstructured hinge

139 domain separating RRM2 from RRM3 (Fig 2A and Supplemental Fig S1A). Given the much 140 more sophisticated tasks associated with the social life of bees, the presence of only single 
141 ELAV in their brains, compared to three pan-neuronally expressed genes in brains of adult flies,

142 was surprising and prompted us to investigate whether elav in bees is alternatively spliced to

143 compensate for the lack of the expected genic diversity. Indeed, cloning of full-length elav from

144 RT-PCR revealed five alternatively spliced exons: exons 3a, exon 4a adding an additional 3’ss,

145 exon 4b adding an additional 5'ss, exon 4c and exon 4d, (Fig 2A-C, Supplemental Fig S1). The

146 combination of these exons in addition to skipping of exon 4 variables potentially generates 38

147 different isoforms (Fig 2A).

Intriguingly, two of these alternative exons are located in the loop region of RRM2 and

149 the other three are located in the hinge region (Fig 2A-C, Supplemental Fig S2A). Exon 4d is

150 only 3 nt long and codes for a serine which potentially can be phosphorylated to impose further

151 control of ELAV function ${ }^{37}$. Since the sequence of exon $4 \mathrm{~d}$ is TAG and flanked by AG/GT

152 consensus splice sites it is not a substrate for recursive splicing ${ }^{40-42}$. Exon $4 \mathrm{~d}$ is too small to

153 accommodate spliceosomal complexes on both sides and must thus be spliced sequentially ${ }^{11}$.

154 Rather unexpectedly, we also detected isoforms which skip exon 4 and its variants

155 encoding the second half of RRM2. This results in truncated ELAV proteins by introducing a

156 frameshift removing much of the beta-sheet of RRM2 involved in RNA recognition as well as

157 alpha-helix 2 that makes up the backbone of the RRM structure. Since skipping of variable exons

158 between exon 3 and 4 deemed unfunctional based on RNA binding assays ${ }^{43}$, we employed

159 molecular modeling to explore the capacity of frequently included alternative exons 3a and 4a to

160 build alternative structures that might hold functionality. Indeed, inclusion of exon 3a with

161 concomitant exclusion of exon 4, 4a and/or $4 \mathrm{~b}$ adds an additional beta-sheet potentially

162 increasing the capacity to bind RNA (Supplemental Fig 2B). Inclusion of exon 4c further adds an

163 additional alpha-helix likely stabilizing this alternative RRM structure (Supplemental Fig 2C). 
Intriguingly, exon 4c from bees has been found conserved in Drosophila FNE and aligns

165

166

167

168

169

170

171

172

174

175

176

177

178

179

180

181

182

183

184

185

to part of ELAV ${ }^{35,36,44}$. Human ELAV/Hu family proteins also harbor an alternatively spliced small exon between the second and third RRM at a position similar to that of the hinge region of bee elav, before a conserved motif involved in nuclear cytoplasmic shuttling ${ }^{45}$. Alignment of exon 4c from bees with orthologues in other insects (the mosquito Aedes aegyptii and the moth Bombyx mori), zebrafish Danio rerio, African clawed frog Xenopus laevis, Chicken Gallus gallus and human ELAV/Hu proteins revealed evolutionary conservation of this microexon (Fig 2D), which is consistent with an evolutionary conserved microexon program between vertebrates and invertebrates ${ }^{46,47}$.

Bees also have a $3 \mathrm{nt}$ microexon (Fig 2A-C), that adds a serine, which potentially can be phosphorylated ${ }^{37}$. In vertebrates, this serine is added through an alternative 3 'splice site at the same position in Human $H u B$ and $H u C$, chicken ELAVL2-4, Xenopus elrB and elrC, and zebrafish ELAVL2 and ELAVL3.

Next, we analyzed alternative splicing in more detail than possible on agarose gels, where multiple alternative splice products, amplified from mRNA of larval brains, where detected only as a smear (Fig 3A). Consistently with the small differences of alternative splice products, alternative protein isoforms were not separable either by Western blot detection (Fig 3B). Therefore, we employed a higher resolution separation of ${ }^{32} \mathrm{P}$-labeled PCR products using denaturing polyacrylamide gels. This analysis revealed 23 distinguishable products with sizes between 78 and 463 nt (Fig 3 C and D). Most frequently found isoforms were 3-4-5, 3-3a-4-4c-5, 3-3a-4-5/3-4-4c-5 and 3-3a-4b-5/3-4b-4c-5 as well as the truncated isoforms 3-4c-5 and 3-5, thus indicating functional relevance for the newly identified alternative splice products. 
Since some of the isoforms were not separable based on size, we wanted to determine

187 how frequently each alternative exon is included. For this purpose, we digested 5, ${ }^{32} \mathrm{P}$-labeled PCR products with KpnI or FokI restriction endonucleases to cleave off their unlabeled 3' parts

189 (Fig 2C, 3E and 3F). For both sides of exon 4, all possible combinations of alternative splice 190 products were detected.

Next, we analyzed the ELAV alternative splicing pattern at different developmental 192 stages and in different tissues (n=3, Fig 3G-I and Supplemental Fig 3). This analysis revealed 193 dynamic inclusion of alternative exons. Most strikingly, splicing from exon 3 to 4 is absent in 194 embryos and skipping of exon 4, 4a or 4b leads to considerably increased abundance of the 195 truncated isoform 3-4c-5 in adults, particularly workers.

196 To obtain further insights into the dynamics of elav alternative exon use at a cellular level 197 we performed whole mount RNA in situ hybridization with anti-sense probes against alternative 198 exons 3a and 4c in brains of worker bees. Most strikingly, both exons 3a and 4c show very 199 dynamic inclusion levels in the mushroom bodies, displaying unique patterns in each individual 200 bee (Fig 4 A, D, J and M). In contrast, inclusion levels in the medulla (visual neuropile not 201 involved in the learning process) are uniform for both isoforms (Fig 4G and P).

203 ELAV protein levels are dynamic in mushroom bodies of worker bees

204 ELAV family proteins are pan-neurally expressed in Drosophila. Their expression seems not to 205 be dynamic as judged form antibody stainings, but changes in nuclear and cytoplasmic 206 distributions have been observed ${ }^{24}$. Consistent with its nuclear localization in flies, ELAV is 207 also mostly nuclear and broadly expressed in about 75\% of worker bee brains analyzed (Fig 5A). 208 In the remaining 25\%, however, ELAV expression was very dynamic, showing patches of 
nuclear and cytoplasmic localization, but also small patches of cells with no ELAV expression

210 (Fig 5D,G, J). Because these analyses were done on animals whose previous experience in the

211 field could not be controlled, we wondered whether such localized changes in ELAV expression

212 might be indicative of experience-dependent plasticity.

214 ELAV expression and alternative splicing is altered upon learning

215 Since bees depend on learning and memory to forage, the pronounced loss of ELAV expression

216 in some of the brains of worker bees might reflect inter-individual learning/memory variations.

217 Thus, we thought of testing if such local down regulation might be indicative of a particular

218 individual learning/memory status. To increase the sensitivity of our follow-up molecular

219 analysis we took advantage of the diversity in speed of learning observed among individuals

220 during a 5-trial training by splitting trained bees into fast and slow learners, e.g. bees that

221 responded in the first two trials and every time after the initial response vs bees with a lack of

222 response in the first two trials or with gaps after the initial response (Fig 6A). We then monitored

223 elav expression levels from their brains by qPCR at various time points after training (Fig 6B).

224 This analysis indeed revealed that elav steady-state mRNA levels had significantly dropped two

225 hours after training in the fast learners compared to slow learners $(p=0.13)$. We therefore thought

226 to analyze alternative splicing of elav exons 3a and 4c two hours after training. Indeed, we

227 detected a significant increase in inclusion of exons 3a and 4c in the mushroom bodies, but not in

228 the medulla one hour after training in fast learners (Fig 6C-E). We also analyzed the alternative

229 splicing pattern of ELAV on denaturing polyacrylamide gels, but no differences were detected

230 after learning in this assay, likely because the observed changes occurred only in relatively few

231 cells (Supplemental Fig 4). 


\section{Discussion}

234 Many RNA binding proteins including neuronal ELAV/Hu RBPs are comprised of families of

235 highly related proteins ${ }^{16,48}$. In case of ELAV family RBPs, they have unique individual

236 functions, but depending on cellular localization and concentrations they can cross-regulate

237 targets making the study of their individual functions difficult ${ }^{24,35,36,43}$. Therefore we took

238 advantage of honeybees due to presence of only a single elav gene to examine whether ELAV is

239 required for learning and memory.

241 A role for ELAV in learning and memory

242 Although neuronal ELAV/Hu family proteins are broadly expressed in the brain, mutants of

243 individual genes in mice and Drosophila revealed only subtle developmental defects thus

244 pointing towards a primary role in regulating neuronal functions as e.g. operating in learning and

245 memory 18,24,49,50. A knock-out of $\mathrm{HuC}$ in mice revealed a role in the synthesis of the

246 neurotransmitter glutamate resulting in reduced neuronal excitability and impaired motor

247 function ${ }^{18}$. For HuD, roles in learning and memory have been suggested due to its involvement

248 in regulating GAP43 expression which has established roles in learning and memory ${ }^{51-53}$. Here,

249 overexpression of $\mathrm{HuD}$, which is cytoplasmic, leads to increased GAP-43 expression by

250 increasing mRNA stability. Since in bees elav steady state mRNA levels drop for a short period

251 after training early during memory consolidation, this might reflect functional

252 compartmentalization of ELAV/Hu family proteins between nucleus and cytoplasm as bee

253 ELAV is mostly nuclear compared to $\mathrm{HuD}$, which is mostly cytoplasmic in a learning context in

254 the mouse hippocampus ${ }^{52}$. 
255 The changes in elav expression in the brain occur within two hours following learning consistent

256 with a role in memory consolidation. Indeed, our learning protocol was designed to trigger the

257 formation of stable long-term memories, which can be detected several days later ${ }^{54}$. Such

258 memories are formed through a consolidation process initiated before the end of training and

259 within a few hours, which depends on gene transcription ${ }^{10,39}$. It is therefore conceivable, that

260 altered levels of ELAV will impact on newly transcribed genes. In particular, expression of

261 ELAV has been linked to implementing splicing programs governing neuronal characteristics

262 such as changes in cell adhesion. Potentially, reduction of elav levels could reduce cell adhesion

263 for facilitating creation or pruning of new synaptic connections. Indeed, changes in connectivity,

264 particularly in the mushroom bodies, is an important process underlying long-term memory

265 formation ${ }^{55}$. Such role is well in agreement with our observations in Drosophila, where reducing

266 alternative splicing of the ELAV target ewg, a transcription factor, results in increased growth of

267 synapses at the NMJ ${ }^{17,56}$. Likewise, we observed changes in ELAV alternative splicing in bees

268 leading to an increase in exon 3a and 4c inclusion, which is anticipated to have profound effects

269 on target mRNA binding. In addition, skipping of exon 4, 4a or 4b leads to a frameshift and an

270 altered structure of the third RRM, which will alter target specificity and/or reduce binding

271 affinity.

272

273 Local and dynamic expression changes of ELAV as a hallmark for its role in learning and

274 memory

275 A hallmark of memory formation is altered local gene expression followed by local changes of

276 neuronal properties and establishment of new connections ${ }^{1,3}$. Activity induced expression of

277 IEG transcription factors has been associated with memory ${ }^{4,5}$. Intriguingly, expression of HuD 
278 can be induced by neuronal activity ${ }^{57}$. Stabilization of C/EBP by apELAV1 in Aplysia 279 accompanies long-term memory ${ }^{58}$, although apELAV1 is mainly nuclear in contrast to 280 apELAV2, which is also cytoplasmic ${ }^{59}$.

281 In agreement with a role for ELAV in memory formation we find variable expression patterns 282 for ELAV in the mushroom bodies of worker bees typical of IEGs. Even more compelling, the 283 expression pattern of ELAV in the mushroom bodies of worker bees is unique and differs 284 between individuals. Similarly, inclusion levels of alternative exons 3a and 4c also show unique 285 patterns in each individual bee. This can be understood as possible consequences of differences 286 in the previous experience that individuals had had, either within the hive or outdoors (e.g. social 287 interactions, environmental stimuli). Indeed, the mushroom body connectivity is shaped by 288 individual experience during a continuous maturation process ${ }^{60,61}$. Yet, molecular tools are 289 currently lacking in the honey bee in order to identify those neurons where elav expression varies 290 and compare establish interindividual comparisons. In addition, ELAV’s cellular localization 291 also varied in individual cells in the mushroom bodies from nuclear to cytoplasmic. Such 292 differences in cellular localization are expected since Drosophila ELAV localizes mostly to the 293 nucleus, RBP9 is cytoplasmic and FNE is found in both compartments. However, ELAV/Hu 294 family proteins also shuttle between nucleus and cytoplasm ${ }^{24,62}$. Upon removal of ELAV in 295 Drosophila, alternatively spliced microexon 4c is included in FNE leading to nuclear localization 296 and regulation of alternative splicing of genes that are otherwise ELAV targets ${ }^{35,36}$ suggesting a 297 complex network of interactions among ELAV/Hu proteins.

298 Alternative splicing could serve as an adaptive mechanism to changes in perception, but also to 299 environmental conditions such as toxic insult ${ }^{63}$. Although learning and memory is affected by 300 neonicotinoids in insects, we did not find any changes in elav alternative splicing ${ }^{44,64,65}$. Since 
301 we also could not detect any alternative splicing changes after learning in mRNA from central

302 brains, drastic changes in alternative splicing relevant to learning and memory might occur only

303 in few cells.

Alternative splicing of a microexon in ELAV/Hu proteins is evolutionary ancient

306 Human HuB-D genes contain an alternatively spliced microexon in the hinge region between the

307 second and third RRM ${ }^{20,66,67}$. Intriguingly, we identified an alternatively spliced exon at the

308 same position in the single bee elav gene ${ }^{44}$. Comparison of the sequence between human and

309 insects of this exon shows a high sequence similarity indicating that this exon is evolutionary

310 ancient. Previously, exon duplication between humans and Drosophila has been documented in

311 few ion channel genes leading to alternatively spliced exons, but with a different sequence and

312 no longer exons are evolutionary conserved between invertebrates and vertebrates ${ }^{46,47}$.

313 Intriguingly, ELAV in Drosophila has lost its introns due to retrotransposition, but retained

314 microexon $4 c^{19}$. This microexon is involved in regulating nuclear localization of ELAV and

315 FNE in Drosophila ${ }^{35,36,45}$, and also affects localization of HuD in human cells ${ }^{66,67}$. Its increased

316 expression shortly after training thus coincides with an initial nuclear role of ELAV at the

317 memory consolidation phase, which requires transcription ${ }^{10,39}$.

318 For most neuronally alternative spliced microexons in mice, Srrm4 is required for their inclusion

$319 \quad 68$. Srrm4 contains a novel evolutionary conserved protein domain 'enhancer of microexons'

320 (eMIC) that is present in Drosophila Srrm2/3/4 and required for exon inclusion in the Dscam

321 exon 9 cluster ${ }^{69}$. Taken together, a conserved neuronal microexon program is present in

322 vertebrates and insects ${ }^{47}$. 


\section{Alternative splicing in bee ELAV is confined to unstructured linker regions, but not RNA}

\section{5 recognition domains}

326 A main question arising from the presence of multiple highly related genes is whether they act in

327 an overlapping manner. In case of Drosophila ELAV family members ELAV, FNE and RBP9 in

328 Drosophila, distinct mutant phenotypes and the lack of major genetic interactions among them

329 suggests largely independent functions ${ }^{24}$. However, cross-regulation between FNE and RBP9 is

330 present in the regulation of synapse numbers. Likewise, expression in non-neuronal cells or

331 swapping of expression and localization regulatory regions can to a large degree substitute for

332 their individual functions and they can cross-regulate. Overlapping functions even extend to

333 more distantly related Sex lethal (Sxl), which is required for neuronal functions in Diptera, but

334 has been recruited in Drosophila for sex determination and dosage compensation ${ }^{70}$. Here, RBP9

335 is required for maternal inhibition of dosage compensation, a function that is taken over entirely

336 by Sxl during embryogenesis ${ }^{24}$.

337 These facts point out that the main distinction among ELAV family members only minimally

338 occurs at the level of RNA recognition. Hence, it is conceivable, that the ELAV family in bees

339 has "merged back" into a single copy gene by incorporating the variable parts between family

340 members by alternative splicing. In this respect, it is very interesting that alternative splicing in

341 bee ELAV occurs in unstructured linker regions between RRMs. It is conceivable, that these

342 regions mediate protein-protein interactions leading to sub-functionalization. Accordingly, the

343 conserved microexon present in the hinge region likely serves such purpose, but the interacting

344 proteins remain to be identified.

345 Mis-regulation of microexons has been found as a major cause of autism spectrum disorders

346 revealing essential functions for such microexons in neurons ${ }^{68}$. Notably, inclusion levels of this 
347 microexon in bees is altered upon learning and memory formation. Hence, lack of dynamic

348 inclusion of microexons in ELAV/Hu family proteins might point towards a role in establishing

349 the extensive memories often associated with some autism spectrum disorders ${ }^{71}$.

$351 \quad$ Materials and Methods

\section{Honey bees and treatment}

353 Honey bees (Apis mellifera) were collected from flowers or local bee hives in the UK for

354 molecular biology experiments (worker bees were used unless otherwise specified). For 355 behavioral experiments, workers were taken from the experimental apiary on the university 356 campus in Toulouse (France), on the morning of each experiment. Following cold-anesthesia, 357 they were harnessed in metal tubes leaving access to the head, fed with $5 \mu$ l of sucrose solution 358 (50\% weight/weight in water) and then kept in the dark at room temperature until needed. They 359 were fed in the same way on every morning and evening during the time of each experiment.

\section{Behavioral assays}

362 Learning and memory capacities were assessed using a standard protocol based on the olfactory 363 conditioning of the proboscis extension response (PER) ${ }^{72}$, which consisted of 3 learning trials

364 (unless specified otherwise) where animals were trained individually to associate an odorant with 365 a sucrose reward as detailed below. Memory of the association was tested either one hour (short366 term memory) or 48 hour (long-term memory) after the last learning trial. In all experiments, 367 bees of both treatment groups were trained in parallel. Each learning trial (40 s) started when the 368 restrained bee was placed in front of an odorless air flow. After $15 \mathrm{~s}$, the setup allowed to deliver 369 an odor (conditioned stimulus, CS) for $4 \mathrm{~s}$ by partially diverting the flow in a syringe containing 
370 a filter paper soaked with $4 \mu \mathrm{l}$ of pure odorant. (1-hexanol and 1-nonanol were used,

371 alternatively for different bees; data were pooled after checking for any significant effect of the

372 odorant used). Sucrose (unconditioned stimulus, US: same solution as for feeding) was delivered

373 to the antennae using a toothpick, $3 \mathrm{~s}$ after CS onset, for $3 \mathrm{~s}$. This triggered the bee’s reflex

374 extension of the proboscis to lick the reward. Whenever the animal already responded to the CS

375 (conditioned response), it was directly allowed to feed upon US onset. Successive learning trials

376 were separated by 10 -min intervals to facilitate memory consolidation ${ }^{54}$. Memory was assessed

377 by placing the animals again in the conditioning setup, and by presenting them the CS without

378 the US ${ }^{72}$. The presence or absence of a conditioned response was recorded. In case of no

379 response, sucrose was applied to the antennae at the end of the test, to control for the intact motor

380 response. Bees failing to show an intact reflex were discarded. Bees that responded to the

381 training in the first two trials and that responded every time were classified as fast learners. Bees

382 that responded only two times in the four trials were classified as slow learners. The sucrose and

383 odorants were purchased from Sigma-Aldrich (France).

385 Recombinant DNA technology, RT-PCR, qPCR and analysis of alternative splicing

386 Recombinant DNA technology was done according to standard procedures as described ${ }^{73}$. Bee

387 elav was amplified from oligo dT primed cDNA made from larval brains using primers elav F1

388 (GCCGCCGGCGCGAACGGAATGGACACAGTCGTACAAC) and elav R1

389 (GCGTCTAGAGGCGCGCCTCTACGCCGCCTTGCTCTTGTTCGTCTTGAAGC) and cloned

390 into a modified pBS SK+ using NgoMIV and XbaI. Clones $(n=45)$ were sequenced using

391 primers elav F1 and elav R1. RNA extraction from whole bees or dissected bee brains and RT-

392 PCR was done as described ${ }^{74}$. elav expression at different timepoints was compared to Appl 
expression using primers AM elav qF3 (CCCTCTTCTCGAGCATTGGCGAGGTTG) and AM

394 elav qR3 (GCCGTACGGGCTGAATAGATTCTCCAG) to amplify the constant part of elav and

395 normalized to unpaired control animals using qPCR as described ${ }^{65}$. For high resolution analysis

396 of elav alternative splicing primers elav F2 (GTCGCGGATACTTTGCGACAACATCAC) and

397 elav R2 (CCCGGGTAGCATCGAGTTTGCCAATAGATC) were used to amplify elav from

398 cDNA. One of the primers was labeled using gamma ${ }^{32} \mathrm{P}-\mathrm{ATP}(\mathrm{NEN})$ and PCR products were

399 separated on sequencing type denaturing polyacrylamide gels. Polyacrylamide gels were dried,

400 exposed to phosphoimager screens (BioRad) and quantified with QuantityOne (BioRad).

401

RNAi, Western analysis, RNA in situ, antibody stainings and imaging

403 For RNAi knockdown in bees, elav and GFP DNA templates for in vitro transcription were 404 amplified for elav from a cloned cDNA with primers Apis ELAV T7 RNAi F1 405 (GGAGCTAATACGACTCACTATAGGGAGAATGATGGCGAACGGAATGGACACAG)

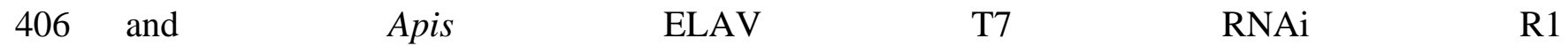

407 (GGAGCTAATACGACTCACTATAGGGAGACTACGCCGCCTTGCTCTTGTTCGTCTTG)

408 and for GFP a 700 bp fragment was amplified with primers GFP T7 RNAi F1 409 (GGAGCTAATACGACTCACTATAGGGAGACTGTTCACCGGGGTGGTGCCCATC) and $\begin{array}{lllll}410 & \text { GFP } & \text { T7 } & \text { RNAi } & \text { R1 }\end{array}$

411 (GGAGCTAATACGACTCACTATAGGGAGACTTGTACAGCTCGTCCATGCCGAGAG).

412 Double stranded RNA was generated by in vitro transcription with T7 polymerase with the 413 MegaScript kit (Ambion) for $3 \mathrm{~h}$ according to the manufacturer's instructions. After digestion of 414 the template with TurboDNAse (Ambion), dsRNA was phenol/chloroform extracted, ethanol 415 precipitated and taken up in RNAse free water at a concentration of $5 \mu \mathrm{g} / \mu \mathrm{l}$. The dsRNA (250 nl) 
416 was then injected into the brain through the median ocellus with a Nanoject II microinjector

417 (Drummond). RNAi efficiency testing for ELAV was done from dissected central brains by

418 Western blotting according to standard protocols as described ${ }^{73}$ using a polyclonal rat anti-

419 ELAV antibody generated against Drosophila ELAV (1:800) ${ }^{45}$ and secondary HRP-coupled 420 goat anti-rat antibody (1:5000, GE Healthcare) by chemoluminescence detection (Pierce)

421 according to the manufacturer’s instructions.

422 Brain antibody stainings were done with rat polyclonal anti-ELAV antibody (1:200) as

423 described ${ }^{17}$ and counterstained with DAPI $(1 \mu \mathrm{g} / \mathrm{ml})$.

424 To make probes for RNA in situ hybridizations, a pBS SK+ vector was modified by

425 cloning a U-rich stem loop at the end of the in vitro transcript using EcoRI and KpnI and

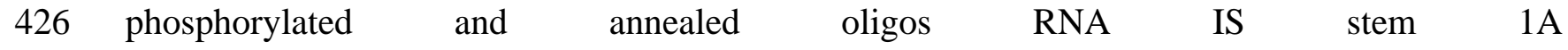

427 (CTCAAACACATATATACATATACATATAGGGGTACATACATATATACATATATACTC

$\begin{array}{llll}428 & \text { GAG) } & \text { B }\end{array}$

429 (AGTATATATGTATATATGTATGTACCCCTATATGTATATGTATATATGTGTTTGAGG

430 TAC), tango13A

431 (TATGGTAAGCCAGATGCATGGGTGCAGGACAACACGTCGAAGttaGATATCG) and B

432 (AATTCGATATCtaaCTTCGACGTGTTGTCCTGCACCCATGCATCTGGCTTACCATACTC

433 G). ELAV alternative exons were then cloned with XhoI and PstI using phosphorylated and

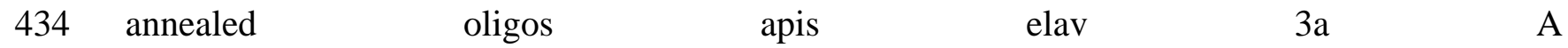

435 (TCGAGACAACATCACCGTACGACAGTTTGTGACCGGCGGCGGAGACTATTTGCCCG

436 GATTGTCGAAAAGTACTGAATTCCTGCA) and B

437 (GGAATTCAGTACTTTTCGACAATCCGGGCAAATAGTCTCCGCCGCCGGTCACAAAC

438 TGTCGTACGGTGATGTGTC) and $\quad$ apis elav $\quad$ 4c $\quad$ A 
442 TTGCCAGTGCTGAAGCGGCC). Vectors were linearized with Acc56I and DIG-dUTP

443 (Roche) labeled anti-sense transcripts were generated by in vitro transcription with T3 RNA

444 polymerase (Ambion) in $10 \mu \mathrm{l}$ from $1 \mu \mathrm{g}$ template DNA. These transcripts were cleaned by 445 centrifugation through a G50 Microspin column (GE Healthcare) in a final volume of $50 \mu \mathrm{l}$. In

446 situ hybridizations were done on whole brains in $50 \%$ formamide buffer as described ${ }^{17}$ using

$447 \quad$ 1:500 diluted probes at $39{ }^{\circ} \mathrm{C}$ for $3 \mathrm{~d}$ and washed overnight in hybridization buffer. DIG-labeled

448 probes were then visualized with an FITC conjugated anti-DIG antibody (Roche) and 449 counterstained with DAPI.

450 For brain imaging, confocal Z stacks were taken using a Leica SP5/SP2, using a 40x-oil

451 objective. For the quantification of stained Kenyon cells, the cross-section equal to the width of

452 the calyx was scanned and the fluorescence intensity quantification was performed as previously

453 described using Image ${ }^{24}$. For the imaging of the calyces of the mushroom bodies of honey bee

454 brains, single optical sections were taken in the x-y plane. The image acquisition settings were 455 kept identical for all preparations.

\section{Protein modeling and statistics}

458 Structure modeling was done using Phyre2 of individual RRMs and assembled into a composite 459 of all three RRMs ${ }^{75}$. Unstructured loop parts in RRMs and the hinge region were inserted 460 manually. 
462 followed by Fisher's protected least significance difference post-hoc test using StatView. To

463 compare proportions of conditioned responses between groups, a repeated-measure analysis of

464 variance (ANOVA) was run for the acquisition data (one factor, treatment, with trial as the 465 repeated measure), and a simple ANOVA for retention data ${ }^{76}$. Post-hoc comparisons of rates of 466 CS-specific responses were done using the Fisher's exact test.

468 Acknowledgments

469 We thank the Winterbourne garden (Birmingham) and Lucie Hotier (Toulouse) for providing

470 bees, Roland Arnold and Reinhard Stöger for discussions and comments on the manuscript. For 471 this work we acknowledge funding from the Sukran Sinan Fund, the Genetics Society, the 472 Biochemical Society and BBSRC. JMD acknowledges funding from the CNRS and Université 473 Paul Sabatier.

\section{Authors' contribution}

476 PU, IUH and MS performed molecular biology experiments, PU, JKG and ND performed 477 behavioral experiments, and JKG performed antibody stainings and in situ hybridization 478 experiments. JMD designed behavioral experiments. MS conceived the project and wrote the 479 original draft of the manuscript. JMD, IUH and all other authors reviewed and edited. M.S. and 480 JMD supervised and acquired funding.

\section{Competing interests}

483 The authors declare that they have no competing interests. 


\section{Data availability}

All data are available in the main text or the supplementary material.

\section{References}

4891 Alberini, C. M. Transcription factors in long-term memory and synaptic plasticity. Physiol Rev 89, 121-145 (2009).

4912 Alberini, C. M., Ghirardi, M., Metz, R. \& Kandel, E. R. C/EBP is an immediate-early gene required for the consolidation of long-term facilitation in Aplysia. Cell 76, 10991114 (1994).

3 Clayton, D. F. The genomic action potential. Neurobiol Learn Mem 74, 185-216 (2000).

4 Sommerlandt, F. M. J., Brockmann, A., Rossler, W. \& Spaethe, J. Immediate early genes in social insects: a tool to identify brain regions involved in complex behaviors and molecular processes underlying neuroplasticity. Cell Mol Life Sci 76, 637-651 (2019).

5 Guzowski, J. F. Insights into immediate-early gene function in hippocampal memory consolidation using antisense oligonucleotide and fluorescent imaging approaches. Hippocampus 12, 86-104 (2002).

6 Minatohara, K., Akiyoshi, M. \& Okuno, H. Role of Immediate-Early Genes in Synaptic Plasticity and Neuronal Ensembles Underlying the Memory Trace. Front Mol Neurosci 8, 78 (2015).

$7 \quad$ Iino, S. et al. Neural activity mapping of bumble bee (Bombus ignitus) brains during foraging flight using immediate early genes. Sci Rep 10, 7887 (2020).

8 Hirano, Y. et al. Shifting transcriptional machinery is required for long-term memory maintenance and modification in Drosophila mushroom bodies. Nat Commun 7, 13471 (2016).

9 Fujita, N. et al. Visualization of neural activity in insect brains using a conserved immediate early gene, Hr38. Curr Biol 23, 2063-2070 (2013).

10 Lefer, D., Perisse, E., Hourcade, B., Sandoz, J. \& Devaud, J. M. Two waves of transcription are required for long-term memory in the honeybee. Learn Mem 20, 29-33 (2012).

11 Soller, M. Pre-messenger RNA processing and its regulation: a genomic perspective. Cell Mol Life Sci 63, 796-819 (2006).

12 Vuong, C. K., Black, D. L. \& Zheng, S. The neurogenetics of alternative splicing. Nat Rev Neurosci 17, 265-281 (2016).

13 Ule, J. \& Blencowe, B. J. Alternative Splicing Regulatory Networks: Functions, Mechanisms, and Evolution. Mol Cell 76, 329-345 (2019).

14 Mirisis, A. A. \& Carew, T. J. The ELAV family of RNA-binding proteins in synaptic plasticity and long-term memory. Neurobiol Learn Mem 161, 143-148 (2019).

15 Perrone-Bizzozero, N. \& Bolognani, F. Role of $\mathrm{HuD}$ and other RNA-binding proteins in neural development and plasticity. J Neurosci Res 68, 121-126 (2002).

16 Soller, M. \& White, K. Elav. Curr Biol 14, R53 (2004). 
52517 Haussmann, I. U., White, K. \& Soller, M. Erect wing regulates synaptic growth in Drosophila by integration of multiple signaling pathways. Genome Biol 9, R73 (2008).

18 Ince-Dunn, G. et al. Neuronal Elav-like (Hu) proteins regulate RNA splicing and abundance to control glutamate levels and neuronal excitability. Neuron 75, 1067-1080 (2012).

53019 Samson, M. L. Rapid functional diversification in the structurally conserved ELAV family of neuronal RNA binding proteins. BMC Genomics 9, 392 (2008).

20 Okano, H. J. \& Darnell, R. B. A hierarchy of Hu RNA binding proteins in developing and adult neurons. J Neurosci 17, 3024-3037 (1997).

21 Kim, Y. J. \& Baker, B. S. The Drosophila gene rbp9 encodes a protein that is a member of a conserved group of putative RNA binding proteins that are nervous system-specific in both flies and humans. J Neurosci 13, 1045-1056 (1993).

22 Samson, M. L. \& Chalvet, F. found in neurons, a third member of the Drosophila elav gene family, encodes a neuronal protein and interacts with elav. Mech Dev 120, 373-383 (2003).

23 Yao, K.-M., Samson, M.-L., Reeves, R. \& White, K. Gene elav of Drosophila melanogaster: a prototype for neuronal-specific RNA binding protein gene family that is conserved in flies and humans. J. Neurobiol. 24, 723-739 (1993). of co-expressed ELAV/Hu proteins control specificity of mRNA processing. Mol Cell Biol 35, 3104-3115 (2015).

25 Mukherjee, N. et al. Integrative regulatory mapping indicates that the RNA-binding protein HuR couples pre-mRNA processing and mRNA stability. Mol Cell 43, 327-339 (2012).

26 Uren, P. J. et al. Genomic analyses of the RNA-binding protein Hu antigen R (HuR) identify a complex network of target genes and novel characteristics of its binding sites. $J$ Biol Chem 286, 37063-37066 (2012). 
34 Rogulja-Ortmann, A. et al. The RNA-binding protein ELAV regulates Hox RNA processing, expression and function within the Drosophila nervous system. Development 141, 2046-2056 (2014).

35 Wei, L. et al. Overlapping Activities of ELAV/Hu Family RNA Binding Proteins Specify the Extended Neuronal 3' UTR Landscape in Drosophila. Mol Cell 80, 140-155 e146 (2020).

36 Carrasco, J. et al. ELAV and FNE Determine Neuronal Transcript Signatures through EXon-Activated Rescue. Mol Cell 80, 156-163 e156 (2020).

37 Brauer, U., Zaharieva, E. \& Soller, M. Regulation of ELAV/Hu RNA-binding proteins by phosphorylation. Biochem Soc Trans 42, 1147-1151 (2014).

38 McNeill, M. S., Kapheim, K. M., Brockmann, A., McGill, T. A. \& Robinson, G. E. Brain regions and molecular pathways responding to food reward type and value in honey bees. Genes Brain Behav 15, 305-317 (2016).

39 Villar, M. E., Marchal, P., Viola, H. \& Giurfa, M. Redefining Single-Trial Memories in the Honeybee. Cell Rep 30, 2603-2613 e2603 (2020).

40 Hatton, A. R., Subramaniam, V. \& Lopez, A. J. Generation of alternative Ultrabithorax isoforms and stepwise removal of a large intron by resplicing at exon-exon junctions. Mol Cell 2, 787-796 (1998).

41 Duff, M. O. et al. Genome-wide identification of zero nucleotide recursive splicing in Drosophila. Nature 521, 376-379 (2015).

42 Sibley, C. R. et al. Recursive splicing in long vertebrate genes. Nature 521, 371-375 (2015).

43 Borgeson, C. D. \& Samson, M. L. Shared RNA-binding sites for interacting members of the Drosophila ELAV family of neuronal proteins. Nucleic Acids Res 33, 6372-6383 (2005).

44 Decio, P. et al. Acute thiamethoxam toxicity in honeybees is not enhanced by common fungicide and herbicide and lacks stress-induced changes in mRNA splicing. Sci Rep 9, 19196 (2019).

45 Yannoni, Y. M. \& White, K. Domain necessary for Drosophila ELAV nuclear localization: function requires nuclear ELAV. J Cell Sci 112, 4501-4512 (1999).

46 Copley, R. R. Evolutionary convergence of alternative splicing in ion channels. Trends Genet 20, 171-176 (2004).

47 Torres-Mendez, A. et al. A novel protein domain in an ancestral splicing factor drove the evolution of neural microexons. Nat Ecol Evol 3, 691-701 (2019).

48 Ray, D. et al. A compendium of RNA-binding motifs for decoding gene regulation. Nature 499, 172-177 (2013).

49 Akamatsu, W. et al. Mammalian ELAV-like neuronal RNA-binding proteins HuB and $\mathrm{HuC}$ promote neuronal development in both the central and the peripheral nervous systems. Proc Natl Acad Sci U S A 96, 9885-9890 (1999).

50 Akamatsu, W. et al. The RNA-binding protein HuD regulates neuronal cell identity and maturation. Proc Natl Acad Sci U S A 102, 4625-4630 (2005).

51 Bolognani, F. et al. Coordinated expression of HuD and GAP-43 in hippocampal dentate granule cells during developmental and adult plasticity. Neurochem Res 32, 2142-2151 (2007). 
61552 Pascale, A. et al. Increase of the RNA-binding protein HuD and posttranscriptional upregulation of the GAP-43 gene during spatial memory. Proc Natl Acad Sci U S A 101, 1217-1222 (2004).

53 Quattrone, A. et al. Posttranscriptional regulation of gene expression in learning by the neuronal ELAV-like mRNA-stabilizing proteins. Proc Natl Acad Sci U S A 98, 1166811673 (2001).

54 Menzel, R., Manz, G., Menzel, R. \& Greggers, U. Massed and spaced learning in honeybees: the role of CS, US, the intertrial interval, and the test interval. Learn Mem $\mathbf{8}$, 198-208 (2001).

55 Hourcade, B., Muenz, T. S., Sandoz, J. C., Rossler, W. \& Devaud, J. M. Long-term memory leads to synaptic reorganization in the mushroom bodies: a memory trace in the insect brain? J Neurosci 30, 6461-6465 (2010).

56 Haussmann, I. U. \& Soller, M. Differential activity of EWG transcription factor isoforms identifies a subset of differentially regulated genes important for synaptic growth regulation. Dev Biol 348, 224-230 (2010).

57 Tiruchinapalli, D. M., Ehlers, M. D. \& Keene, J. D. Activity-dependent expression of RNA binding protein $\mathrm{HuD}$ and its association with mRNAs in neurons. RNA Biol 5, 157168 (2008).

58 Mirisis, A. A., Kopec, A. M. \& Carew, T. J. ELAV proteins bind and stabilize C/EBP mRNA in the induction of long-term memory in Aplysia. J Neurosci (2020).

59 Yim, S. J. et al. Regulation of ApC/EBP mRNA by the Aplysia AU-rich element-binding protein, ApELAV, and its effects on 5-hydroxytryptamine-induced long-term facilitation. J Neurochem 98, 420-429 (2006).

60 Cabirol, A., Brooks, R., Groh, C., Barron, A. B. \& Devaud, J. M. Experience during early adulthood shapes the learning capacities and the number of synaptic boutons in the mushroom bodies of honey bees (Apis mellifera). Learn Mem 24, 557-562 (2017).

61 Cabirol, A., Cope, A. J., Barron, A. B. \& Devaud, J. M. Relationship between brain plasticity, learning and foraging performance in honey bees. PLoS One 13, e0196749 (2018).

62 Fan, X. C. \& Steitz, J. A. Overexpression of HuR, a nuclear-cytoplasmic shuttling protein, increases the in vivo stability of ARE-containing mRNAs. Embo $J$ 17, 34483460 (1998).

63 Zaharieva, E., Chipman, J. K. \& Soller, M. Alternative splicing interference by xenobiotics. Toxicology 296, 1-12 (2012).

64 Tasman, K., Hidalgo, S., Zhu, B., Rands, S. A. \& Hodge, J. J. L. Neonicotinoids disrupt memory, circadian behaviour and sleep. Sci Rep 11, 2061 (2021).

65 Decio, P. et al. Thiamethoxam exposure deregulates short ORF gene expression in the honey bee and compromises immune response to bacteria. Sci Rep 11, 1489 (2021).

66 Wang, H., Molfenter, J., Zhu, H. \& Lou, H. Promotion of exon 6 inclusion in HuD premRNA by Hu protein family members. Nucleic Acids Res 38, 3760-3770 (2010).

67 Hayashi, S., Yano, M., Igarashi, M., Okano, H. J. \& Okano, H. Alternative role of HuD splicing variants in neuronal differentiation. J Neurosci Res 93, 399-409 (2015).

68 Irimia, M. et al. A highly conserved program of neuronal microexons is misregulated in autistic brains. Cell 159, 1511-1523 (2014).

69 Ustaoglu, P. et al. Srrm234, but not canonical SR and hnRNP proteins, drive inclusion of Dscam exon 9 variable exons. RNA 25, 1353-1365 (2019). 
$66170 \quad$ Schutt, C. \& Nothiger, R. Structure, function and evolution of sex-determining systems in

662 Dipteran insects. Development 127, 667-677 (2000).

66371 Gonatopoulos-Pournatzis, T. \& Blencowe, B. J. Microexons: at the nexus of nervous

72 Matsumoto, Y., Menzel, R., Sandoz, J. C. \& Giurfa, M. Revisiting olfactory classical

73 Soller, M. \& White, K. ELAV multimerizes on conserved AU4-6 motifs important for

74 Koushika, S. P., Soller, M., DeSimone, S. M., Daub, D. M.

74 Koushika, S. P., Soller, M., DeSimone, S. M., Daub, D. M. \& White, K. Differential and inefficient splicing of a broadly expressed Drosophila erect wing transcript results in tissue-specific enrichment of the vital EWG protein isoform. Mol Cell Biol 19, 39984007. (1999).

75 Kelley, L. A., Mezulis, S., Yates, C. M., Wass, M. N. \& Sternberg, M. J. The Phyre2 web portal for protein modeling, prediction and analysis. Nat Protoc 10, 845-858 (2015).

76 Mota, T. \& Giurfa, M. Multiple reversal olfactory learning in honeybees. Front Behav Neurosci 4 (2010).

\section{$680 \quad$ Figure legends}

681 Figure 1. Fig 1: ELAV is required for learning and memory consolidation.

682 (A) Western blot detecting ELAV in bee central brains of control GFP and elav dsRNA injected workers $50 \mathrm{~h}$ after injection.

684 (B) Schematic of the treatment to test for ELAV's role in learning.

685 (C) Learning (left) and memory (right) performances of control GFP dsRNA (white, n=66) and 686 elav dsRNA (black, $\mathrm{n}=74$ ) injected worker bees. CS: conditioned odor, $N$ : novel odorant. *: $687 \quad \mathrm{p}<0.05 ; * *: \mathrm{p}<0.01$.

688 (D) Schematic of the treatment to test for ELAV's role in memory consolidation.

689 (E) Learning (left) and memory (right) performances of control GFP dsRNA (white, n=74) and 690 elav dsRNA (black, $\mathrm{n}=77$ ) injected worker bees. $C S$ : conditioned stimulus, $N$ : novel odorant **: $691 \mathrm{p}<0.01 ; * * *: \mathrm{p}<0.001$.

692 (F) Schematic of the treatment to test for ELAV's role in memory retrieval. 
693 (G) Learning (left) and memory (right) performances of control GFP dsRNA (white, n=53) and

694 elav dsRNA (black, $\mathrm{n}=50$ ) injected worker bees. CS: conditioned stimulus, $N$ : novel odorant *:

$695 \quad \mathrm{p}<0.05$.

696

697 Figure 2. Single Bee elav gene is alternatively spliced.

698 (A) Gene model of Apis mellifera elav gene depicting exons (boxes) and their splicing. In total,

69938 different alternative splice products are possible.

700 (B) Length of alternative microexons.

701 (C) Sequence of alternative microexons.

702 (D) Alignment of alternative microexon 4c from bees with a part present in Drosophila ELAV or

703 alternatively spliced microexons in ELAV/Hu family proteins of other species. Note that Apis

704 ELAV microexon 4c is the first alternatively spliced exon shown to be conserved between

705 vertebrates and insects.

706

707 Figure 3. elav alternative splicing is dynamic during development.

708 (A) Multiple products are detected from RT-PCR of RNA from larval brain in the alternatively

709 spliced part of bee elav on an agarose gel. M: Marker

710 (B) Western blot of bee ELAV in larval brains.

711 (C and D) Top and bottom part of a representative $5 \%$ denaturing polyacrylamide gel separating

$712 \quad{ }^{32}$ P-labeled alternative splice products from larval brains. Length of PCR products from splice

713 variants are indicated at the right in bold for prominent products and in italics for very rare

714 products. Vertical lines at the right of indicated splice variants indicate inseparable products. M:

715 Marker. 
716 (E and F) Analysis of alternative splicing from larval brains proximal (KpnI) and distal (FokI) of

717 exon 4 from ${ }^{32}$ P-labeled labeled forward (E) or return (F) primer after digestion with either KpnI

718 or FokI on a representative $5 \%$ denaturing polyacrylamide gels. Length of PCR products from

719 splice variants are indicated at the right in bold for prominent products and in italics for very rare

720 products. M: Marker.

721 (G-I) Developmental and sex-specific alternative splicing of bee elav quantified from denaturing

722 polyacrylamide gels shown as mean with the standard error from three replicates as percent from

723 all splice products from top (G left) and bottom (G right) gel parts and after KpnI or FokI

724 digestion as above from embryos (yellow), larval brains (green), drone brains (light blue),

725 worker brains (dark blue) and queen brains (black). (G and H) \#: The 120 nt product can be

726 either 3, 4c, 5 or 3, 3a, 4d, 5. (I) ^: The 123 nt products are either 4b, 4c, 4d, 5 or 3, 4c, 4d, $5 . *$ :

727 The $120 \mathrm{nt}$ products are either 4b, 4c, 5 or 3, 4c, 5 or 3, 3a, 4d, 5. ${ }^{+}$: The 80 nt products are either

$7284 \mathrm{~b}, 5$ or $3,5+/-4 \mathrm{~d}$.

729

Figure 4. Alternative splicing of elav exons 3a and 4c is dynamic in mushroom bodies.

731 Representative RNA in situ hybridizations in worker bees against elav exon 3a (A, D and G) and

732 exon 4c (J, M and P) in mushroom bodies (A, D, J and M) and the medulla ( $\mathrm{G}$ and P)

733 counterstained with DAPI to visualize nuclei (B, E, H, K, N and Q) and merged pictures (C, F, I,

$734 \mathrm{~L}, \mathrm{O}$ and $\mathrm{R})$. Scale bar in $\mathrm{R}$ is $30 \mu \mathrm{m}$.

735

736 Figure 5. Localization and expression levels of ELAV are dynamic in mushroom bodies.

737 Representative anti-ELAV antibody stainings in worker bees of mushbodies (A, D and G)

738 counterstained with DAPI to visualize nuclei (B, E and H) and merged pictures (C, F and I). 
739 Insets show higher magnifications of ubiquitous expression and nuclear localization (inset in A-

740 C), patchy expression with mostly cytoplasmic localization (inset in D-F) and patchy expression

741 with nuclear and cytoplasmic expression (inset in G-I, arrowhead in G and I). The asterisk in G

742 and I indicates nuclear localization in the lower part of the mushroom body. A summary of

743 ELAV localization in mushroom bodies of worker bees is shown in $\mathrm{J}, \mathrm{n}=20$. Scale bars are 30

$744 \mu \mathrm{m}$ in I and $6 \mu \mathrm{m}$ in the inset.

745

$746 \quad$ Figure 6. elav alternative splicing and expression levels change upon learning.

747 (A) Learning response of bees grouped into fast and slow learners (fast learners $n=10$ and slow

748 learners $n=8)$.

749 (B) elav mRNA expression levels determined by qPCR 0h, $2 \mathrm{~h}$ and $4 \mathrm{~h}$ after training shown as

750 mean with the standard error from two to four replicate central brains normalized to Appl

751 expression $(\mathrm{p}=0.13)$.

752 (C) Inclusion levels of elav exons 3a and 4c quantified from RNA in situ hybridizations from

753 control (unpaired) and fast learning (paired) bees in the mushroom body and the medulla

$754 \quad(\mathrm{p}<0.05, \mathrm{n}=7)$.

755 (D and E) Representative RNA in situ hybridizations against exon 3c in mushroom bodies of

756 control (D) and fast learning (E) bees $2 \mathrm{~h}$ after training. Scale bars are $30 \mu \mathrm{m}$ in $\mathrm{E}$ and $6 \mu \mathrm{m}$ in the 757 inset.

758

759 Supplemental Figure 1. Sequence comparison of ELAV family proteins of bees and 760 Drosophila. 
761 (A) Alignment of single Apis mellifera ELAV with the three Drosophila melanogaster 762 orthologues ELAV, FNE and RBP9. Long and short lines on top indicate the three RRMs and

763 RNP motives, respectively. Black triangles depict intron positions. Alternative exons 3a, 4a, 4c

764 and 4d are indicated at relevant intron positions. The hatched line indicates the sequence deleted

765 in exon $4 \mathrm{~b}$ by alternative splicing. Note that skipping of exons 4 , $4 \mathrm{a}$ or $4 \mathrm{~b}$ will result in a

766 truncated protein due to a frameshift (fs).

767 (B) Phylogenetic tree of Apis and Drosophila ELAV family proteins.

770 Supplemental Figure 2. Structural models of bee ELAV depicting the position of alternative 771 exons.

772 (A) Full length ELAV. Note that alternative exons are in flexible linker regions in RRM2 (exons

773 3a and 4a) and the hinge region (exons 4b, 4c and 4d).

774 (B) Truncated ELAV resulting from skipping of exon 4.

775 (C) Truncated ELAV resulting from inclusion of exon 3a and skipping of exon 4. Note that exon 776 3a adds an additional B-sheet to the truncated second RRM potentially increasing RNA binding.

777 (D) Truncated ELAV resulting from inclusion of exons 3a and 4C, and skipping of exon 4. Note 778 that exon 4c adds a second alpha-helix to the truncated second RRM.

779

780 Supplemental Figure 3. Representative gels from the developmental analysis of ELAV 781 alternative splicing quantified in Fig 3G-I.

782 (A and B) Top and bottom gel part of a representative $5 \%$ denaturing polyacrylamide gel

783 separating ${ }^{32} \mathrm{P}$-labeled alternative splice products from indicated developmental stages on top. 
784 Length of PCR products from splice variants are indicated at the right. \# The 120 nt product can

785 be either 3, 4c, 5 or 3, 3a, 4d, 5. M: Marker.

786 (C and D) Analysis of alternative splicing from indicated developmental stages proximal (KpnI)

787 and distal (FokI) of exon 4 from ${ }^{32}$ P-labeled labeled forward (C) or return (D) primer after

788 digestion with either KpnI or FokI on a representative $5 \%$ denaturing polyacrylamide gels. $\wedge$ The

789123 nt products are either $3,4 c, 4 d, 5$ or $4 b, 4 c, 4 d, 5{ }^{\wedge}$ The 120 nt products are either 3 , 3a, 4d,

7905 or 4b, 4c, 5 or 3, 4c, 5. * The 78/80 nt products are either 4b, 5 or 3, 5 +/- 4d. M: Marker.

791

792 Supplemental Figure 4. Representative gels of ELAV alternative splicing after learning.

793 (A and B) Top and bottom gel part of a representative $5 \%$ denaturing polyacrylamide gel

794 separating ${ }^{32} \mathrm{P}$-labeled alternative splice products from naïve and trained bees from unpaired and

795 paired conditioning of conditioned (sugar) and unconditioned (odor) stimuli at the indicated time

796 after training. Length of PCR products from splice variants are indicated at the right. \# The 120

797 nt product can be either 3, 4c, 5 or 3, 3a, 4d, 5. M: Marker.

798 (C and D) Analysis of alternative splicing from naïve and trained bees from unpaired and paired 799 conditioning of conditioned (sugar) and unconditioned (odor) stimuli at the indicated time after 800 training proximal (KpnI) and distal (FokI) of exon 4 from ${ }^{32}$ P-labeled labeled forward (C) or 801 return (D) primer after digestion with either KpnI or FokI on a representative 5 \% denaturing 802 polyacrylamide gels. $\wedge$ The $123 \mathrm{nt}$ products are either $3,4 \mathrm{c}, 4 \mathrm{~d}, 5$ or 4b, 4c, 4d, $5 .{ }^{\wedge}$ The $120 \mathrm{nt}$ 803 products are either 3, 3a, 4d, 5 or 4b, 4c, 5 or 3, 4c, 5. * The 78/80 nt products are either 4 b, 5 or 804 3, 5 +/- 4d. M: Marker. 
Figure 1

\section{A}

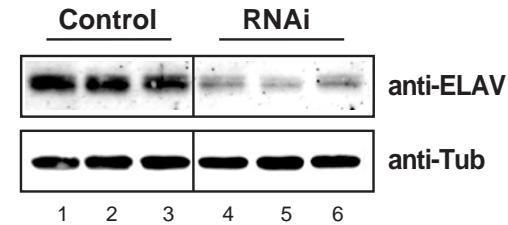

D

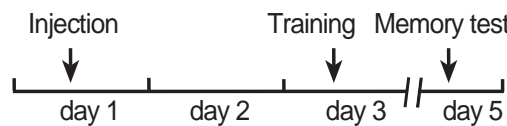

E

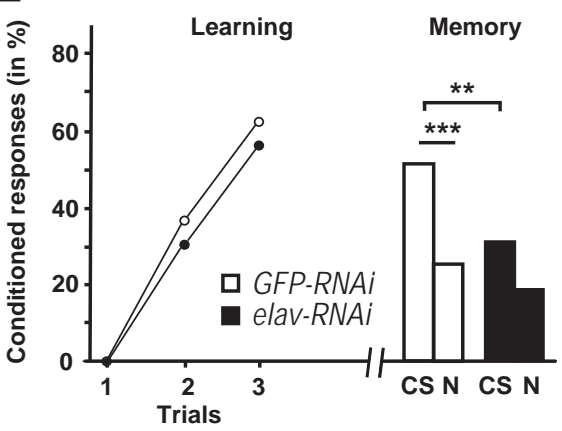

B

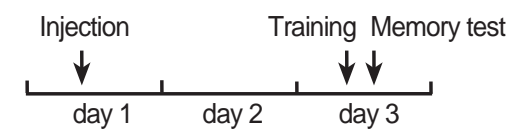

C.

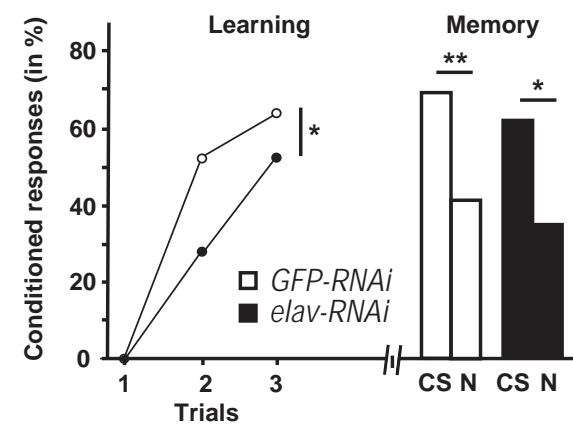

$\mathbf{F}$

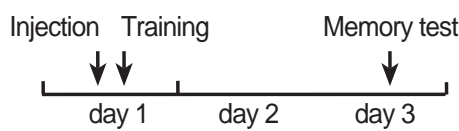

G

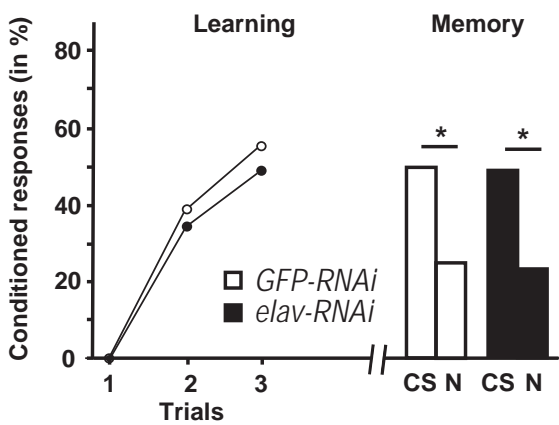




\section{Figure 2}

A

$3 a 4 a \quad 4 b * 4 c \quad 4 d$

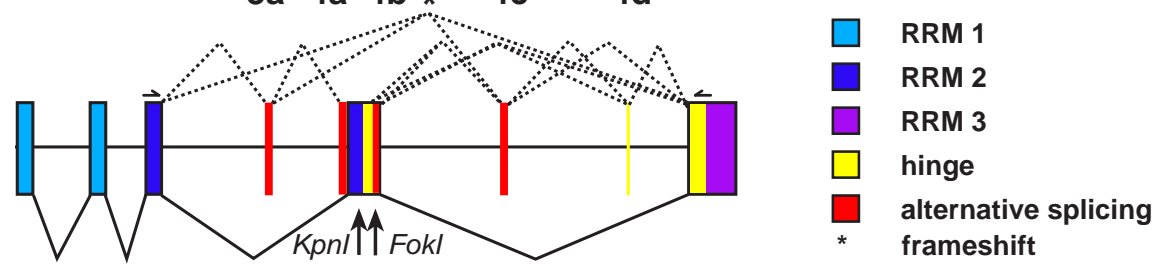

123

4

5

B

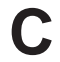

3a: $39 \mathrm{nt}$

4: $256 \mathrm{nt}$

4a: $304 \mathrm{nt}(+48 \mathrm{nt})$

4b: $229 \mathrm{nt}(-27 \mathrm{nt})$

4c: $42 \mathrm{nt}$

4d: 3 nt (recursive splicing)

D
3a: VRQFVTGGGDYLPG ( $E$ with 4a)
4a: EKMMMDPLNNLNRLPTG
4b: $R$ YIPLSPLS $R$
4c: STGKAMLAINKGLQR
4d: $S R$ (adds Ser)
3.5 (4 skipped results in frameshift):
GTARWQAIYWQTRCYPGTR
3, 4c, 5 (4 skipped results in frameshift):
ALARPCLPLTKAYRGTARWQAIYWQTRCYPGTR

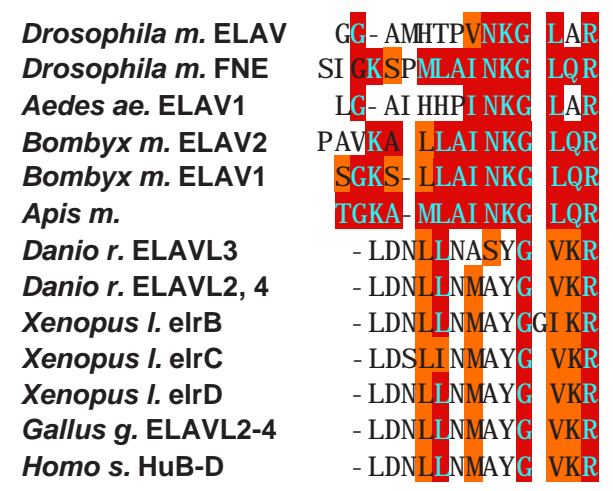




\section{Figure 3}
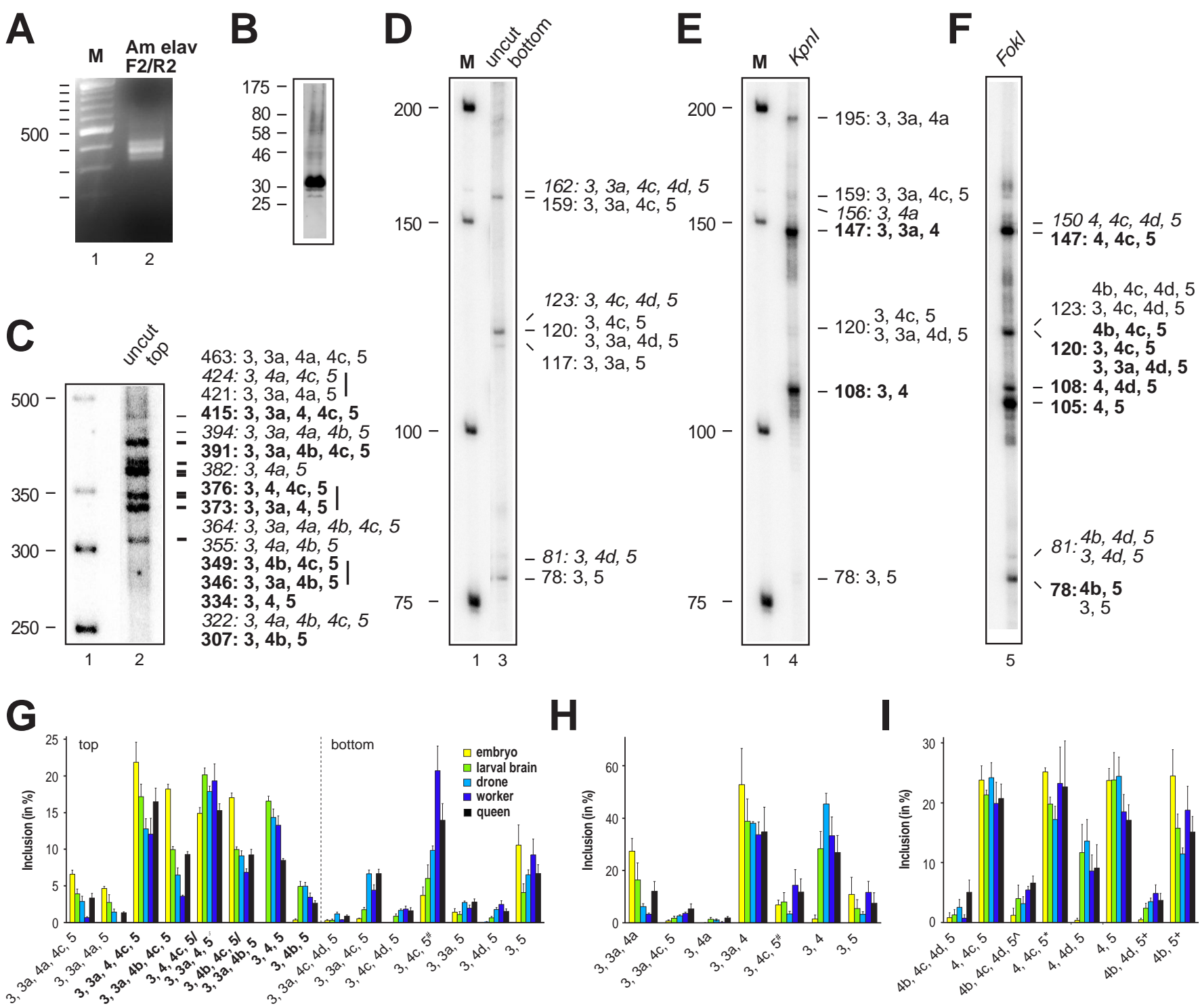


\section{Figure 4}

Exon 3a

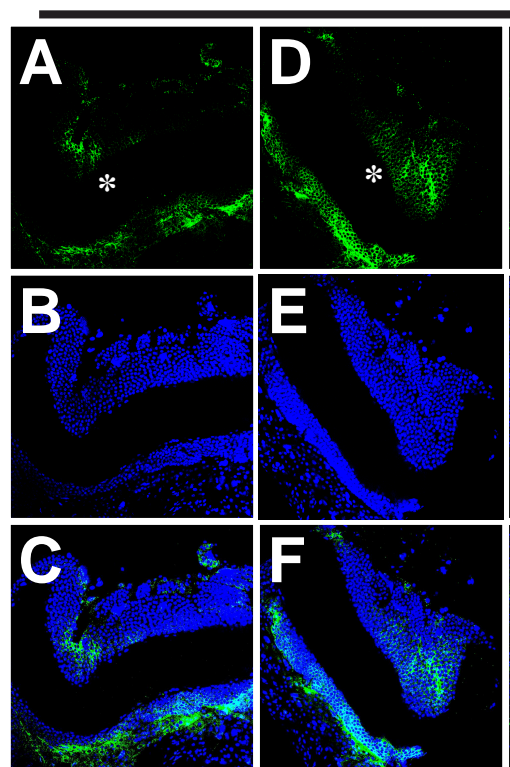

Mushroom bodies

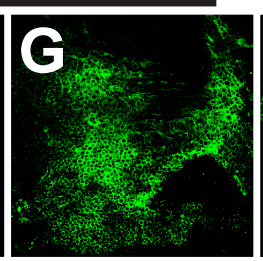

$\mathbf{H}$
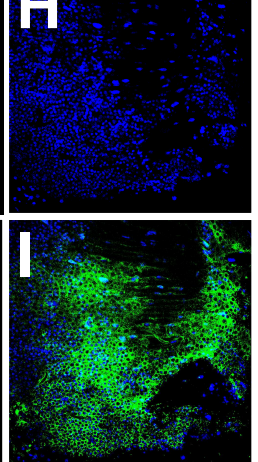

Medulla
Exon 4c

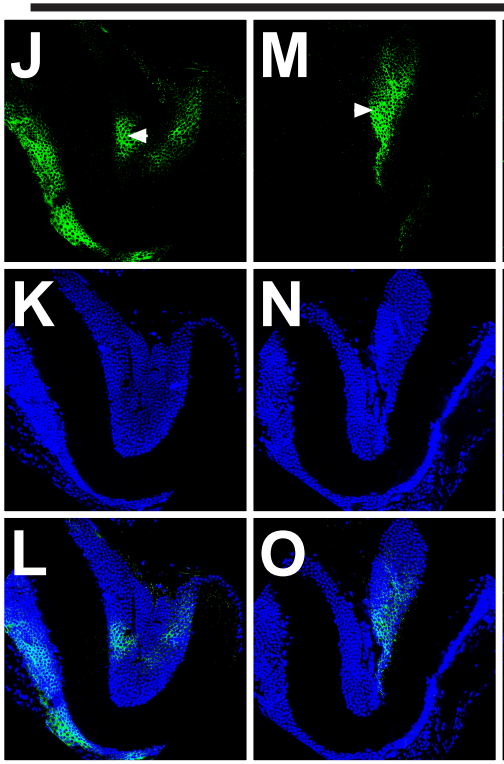

Mushroom bodies

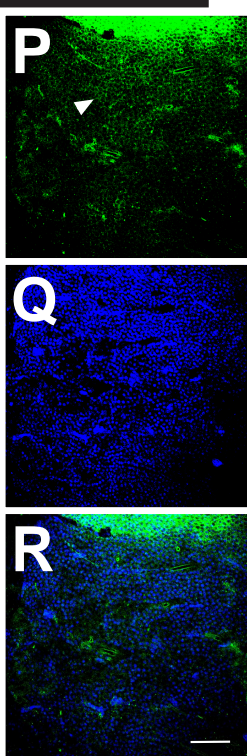

Medulla 
bioRxiv preprint doi: https://doi.org/10.1101/2021.06.24.449637; this version posted June 24, 2021. The copyright holder for this preprint (which was not certified by peer review) is the author/funder, who has granted bioRxiv a license to display the preprint in perpetuity. It is made available under aCC-BY-NC-ND 4.0 International license.

\section{Figure 5}

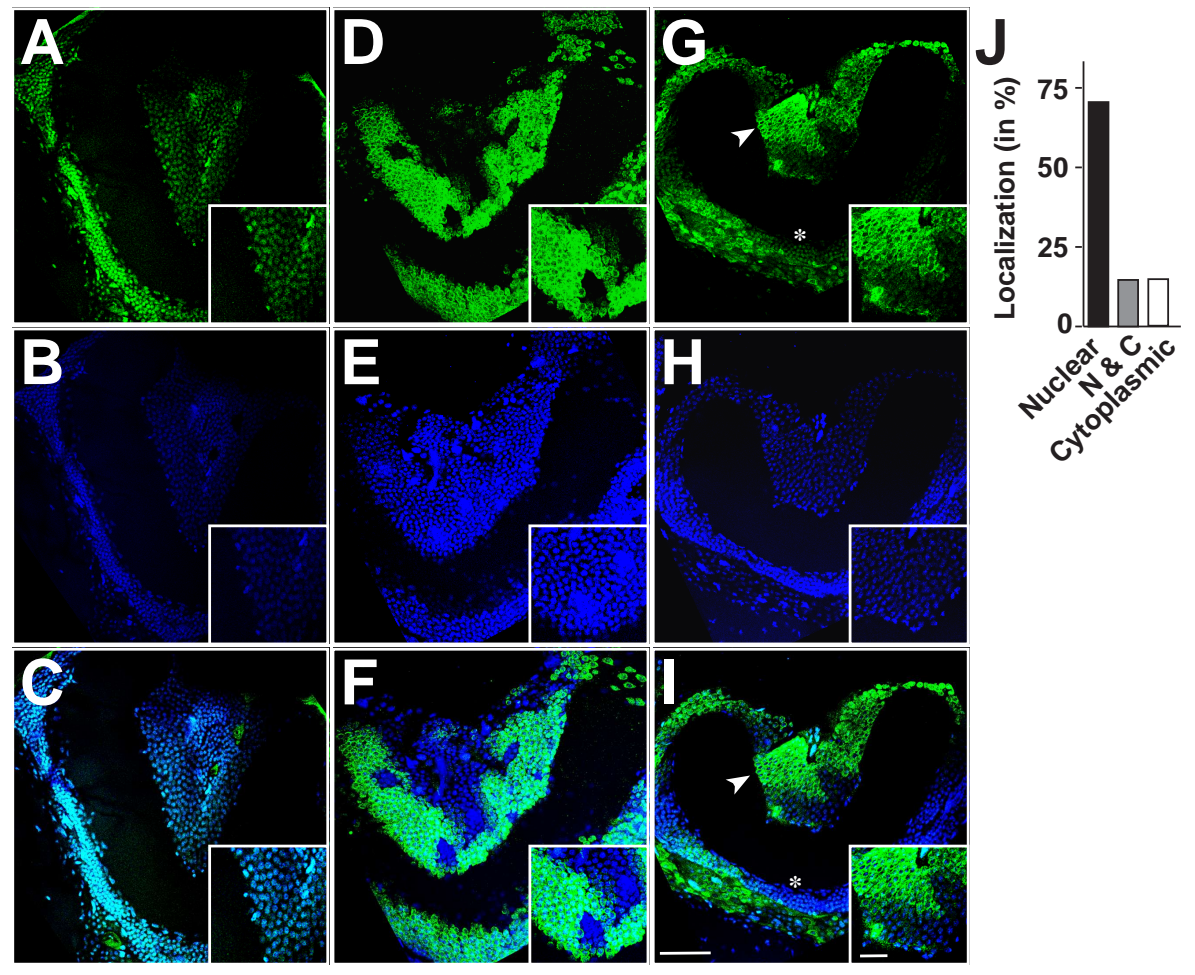




\section{Figure 6}

A

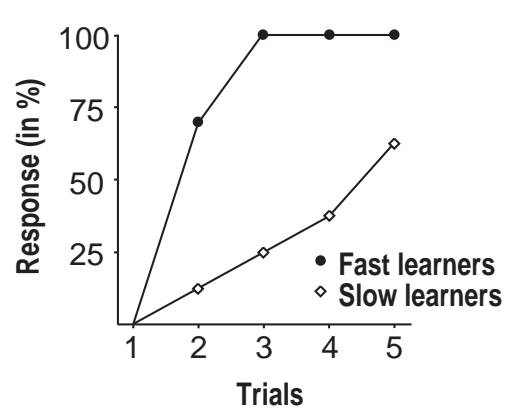

C

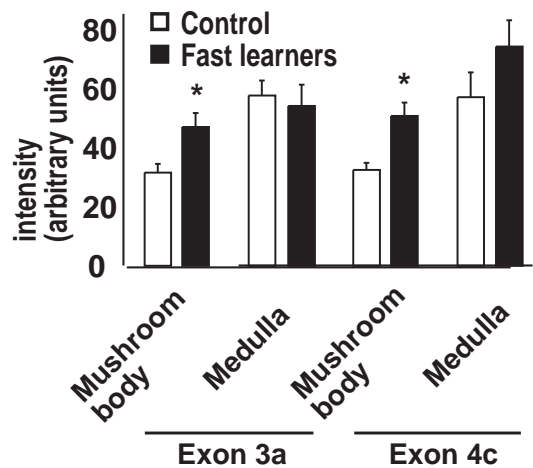

B
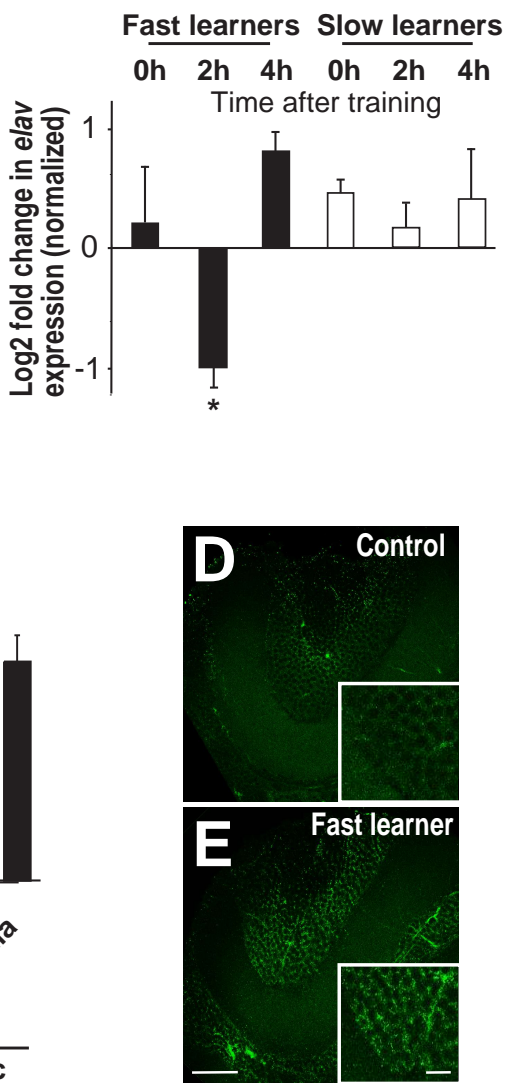